\title{
Surface-based automatic coarse registration of head scans
}

\author{
Fang $\mathrm{Li}^{\mathrm{a}, \mathrm{b}}$ and Zhijian Song ${ }^{\mathrm{a}, \mathrm{b}, *}$ \\ ${ }^{a}$ Digital Medical Research Center, Fudan University, Shanghai 200032, China \\ ${ }^{b}$ Key Laboratory of Medical Imaging Computing and Computer Assisted Intervention of Shanghai, \\ Shanghai 200032, China
}

\begin{abstract}
Surface registration is widely used in image-guided neurosurgery to achieve spatial registration between the patient space and the image space. Coarse registration, followed by fine registration, is an important premise to ensure the robustness and efficiency of surface registration. In this paper, a coarse registration algorithm based on the principal axes is proposed to achieve this goal. The extraction of the principal axes relies on the approximated surface with an adaptive Gaussian kernel, the width of which is consistent with neighborhood relation so that it is applicable for various scanning data. Determining the corresponding centers of translation is another problem for aligning different scanning data, which is solved through heuristics. Six pairs of points on two surfaces with the farthest projections on the principal axes were regarded as the candidates of translation centers, and then through tentative alignments of local regions around them, a pair of candidates with the minimum registration error was selected as the optimal translation centers. Automatic registration of two scans of a head phantom is presented in this paper. Experimental results confirmed the robustness of the algorithm and its feasibility in clinical applications.
\end{abstract}

Keywords: Image-guided neurosurgery, coarse registration, principal axes, adaptive Gaussian kernel, heuristics

\section{Introduction}

Surface-based registration, which is an alternative to point-based registration [1,2] in terms of its noninvasiveness and automation, is a key technology of image-guided neurosurgery [3]. The aim of surface registration is to establish a spatial transformation between the preoperative image space and the intra-operative patient space according to correspondences in two point sets of the patient's head surface. In clinical applications, the two point sets to be registered are usually acquired with different devices, such as computed tomography (CT) and 3D laser scanners, which make it difficult to find point-to-point correspondences between them, and registration is usually achieved by combining coarse registration with fine registration. Fine registration has been successfully solved in existing research work, including the commonly used iterative closest point (ICP) algorithm and its variants $[4,5]$. However, coarse registration is usually domain-dependent and has not been completely solved in robustness and efficiency in the application domain of image-guided neurosurgery.

*Corresponding author: Zhijian Song, Key Laboratory of Medical Imaging Computing and Computer Assisted Intervention of Shanghai, Shanghai 200032, China. Tel.: 86-21-54237054; Fax: 86-21-54237797; E-mail: zjsong@fudan.edu.cn.

0959-2989/14/\$27.50 @ 2014 - IOS Press and the authors. 


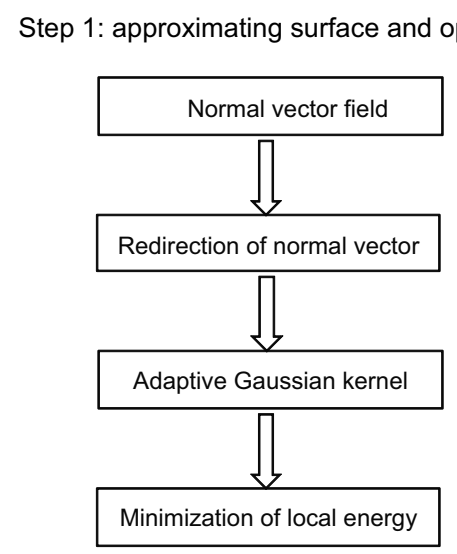

Step 2: estimation of rotation and translation

Fig. 1. Schematic of registration algorithm based on the principal axes of point sets.

Regarding the coarse registration of two scanning point sets, some approaches $[6,7]$ require semiautomatically determined point correspondences of anatomical landmarks on two surfaces, where the registration accuracy highly depends on feature recognition algorithms and is easily affected by the quality of the scanned data. Furthermore, the distribution characteristics of anatomical landmarks on the head make the registration accuracy inconsistent from frontal areas to occipital areas. Since discrete feature matching is not robust enough to align different scans of the head, global similarity was developed as a potentially reliable registration in clinical applications, although most of the work is in the theoretical study phase. For instance, coherent point draft (CPD) [8], as a probabilistic method, models the distribution of a point set with a Gaussian mixture model and its centroid is shifted coherently in a global pattern to preserve the topological structure of the point set. CPD could be well applied in automatic coarse registration; however, its efficiency is low due to the computation in highdimensional space.

Surface registration in image-guided surgery is generally required to be implemented efficiently and conveniently. Therefore, dimension reduction algorithms for coarse registration are more feasible in clinical applications, such as the principal component analysis (PCA-) based method for 2D to 3D image registration [9] and the spin image based registration [10]. In spin image representation, 3D information of the neighboring surface points is mapped to a 2D snapshot of the global shape, so that surface registration can be easily solved by a $2 \mathrm{D}$ correlation between their respective spin images. Similar to the spin image, the principal axes [11] can be used as surface descriptors of dimension reduction. In this paper, a robust coarse registration algorithm based on the principal axes is proposed. Considering the impact of different scanning modes on coarse registration, the principal axes are extracted from an approximated surface, which is optimized with an adaptive Gaussian kernel of point sets, and then six pairs of points on two surfaces with the farthest projections on the principal axes are obtained. Through heuristics, one set of points with minimum registration errors around their local regions will be chosen as the optimal translation centers.

\section{Proposed registration algorithm}

The basic procedure of the algorithm was divided into the following steps: First, the approximated surface was estimated from the original point sets and was optimized. Second, the principal axes were 
extracted from the surface and the corresponding centers of translation were determined through heuristics. Finally, two point sets were aligned according to the rotation and translation transformation. The first two steps are shown in Figure 1.

\subsection{Optimization of approximated surface}

For two point sets obtained with different devices, the principal axes have to be extracted from the approximated surface instead of from the original point sets, because surface approximation can reduce the impact of the scanning mode and noise. The detailed approximating method is described as follows. Given a statistical analysis on the neighborhood of each point, the normal vector field is calculated through eigenvalue decomposition of the covariance matrix of points. Since the directions for calculated normal vectors are not uniform, pointing to exterior or interior of the surface, their redirections have to be performed in such a way that all of the normal vectors point to the outer portion of the surface.

In order to obtain the approximated surface of the point set, moving least squares (MLS) was used to determine the projection of a point along the direction of its normal vector by minimizing the energy function and obtaining a smooth surface as a local approximation of the point set [12]. Given a point $p$, its projection $p_{g}$ is determined by a minimization of the local energy function:

$$
\arg \min \sum\left\|p-p_{g}\right\|^{2} \theta(\|p-q\|)
$$

where $p_{g}$ is the projection of point $p$ along the direction of its normal vector, $q$ is the neighboring point of $p$ and $\theta(\|p-q\|)$ is the weighting function of the neighborhood of point $p$, which is expressed as:

$$
\theta(s)=e^{-s^{2} / h^{2}}
$$

This Gaussian weighting of the local neighborhood is the only factor that can be adjusted in MLS, where $h$ is the width of the Gaussian kernel.

An analysis on the Gaussian weighting revealed that the local approximation with the uniform Gaussian kernel was only suitable for the point sets of the same scanning mode. However, in a clinical practice for two point sets acquired with different devices, there are larger differences in their neighborhood relations; therefore, an adaptive Gaussian kernel is proposed in this algorithm to adapt to various scanning modes, which is realized by a statistical analysis on the local densities of the scanning data. The width of the adaptive Gaussian kernel on point $p$ is calculated by the ratio of the number of its neighboring points within a given region to the radius $r$ of the region:

$$
h(p)=\frac{\text { num }_{p}}{r}
$$




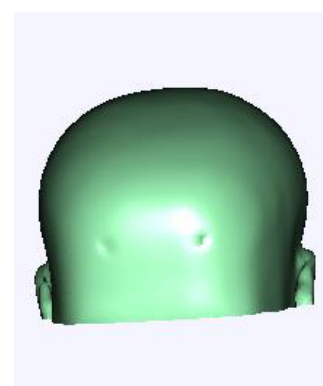

(a)

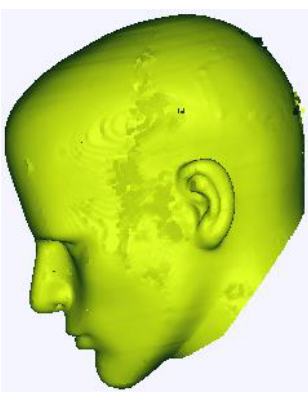

(b)

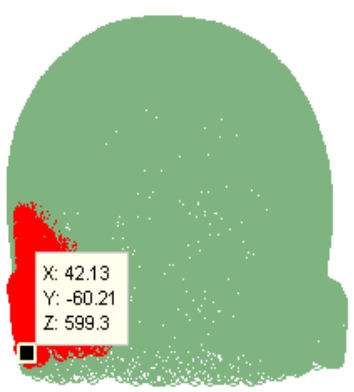

(c)

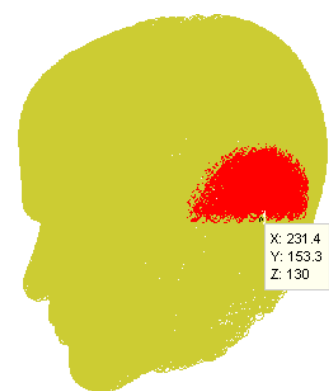

(d)

Fig. 2. A pair of translation centers obtained from two scans. (a) 3D laser scan. (b) CT scan. (c) Translation center on laser scan. (d) Corresponding translation center on CT scan.

where num ${ }_{p}$ is the number of neighboring points on point $p$ and $h(p)$ is the width of the adaptive Gaussian kernel on point $p$.

\subsection{Decision on the corresponding centers of translation}

Through principal component analysis on the approximated surface, the principal axes can be extracted and expressed as three orthogonal vectors.

For two point sets of different scanning modes, the corresponding centers of translation are not easy to obtain from the original point sets, especially for incomplete data. Therefore, the proposed method projects the approximated surface onto the principal axes and finds the six farthest projections on them. Accordingly, the six pairs of points on two surfaces with the farthest projections are regarded as the candidates for translation centers. In order to make a decision on which pair is the optimal center of translation, heuristics are adopted by hypothesizing each pair of candidates as the translation center and aligning the sub-regions around them. From the six alignment results, the pair of the candidates whose sub-regions have minimal registration errors is selected as the optimal centers of translation.

Two scans of a head phantom are shown in Figure 2. Figure 2(a) simulates the laser scan of an incomplete head phantom due to the limitation of surgery, and Figure 2(b) shows the CT scan. The corresponding centers of translation were determined by the proposed method. In Figures 2(c) and 2(d), the optimal pair of center is labeled with their coordinates, whose red local regions are aligned with the minimum registration error. As seen in Figure 2, the optimal corresponding center is located at the ear edge of the head phantom, which is the featured region in the incomplete scan.

\section{Experimental results and discussion}

Two scanning data of a head phantom were used in the experiments, as shown in Figure 3.

\subsection{Robustness of the algorithm}

In order to measure the robustness of the proposed algorithm in clinical practice, $\mathrm{CT}$ scans and 3D laser scans with white Gaussian noise were registered, having noise levels of $0.001 \mathrm{~dB}$ to $0.009 \mathrm{~dB}$. The registration error was calculated from the root mean square error between the two point sets. The previous algorithm using a uniform Gaussian kernel and the proposed algorithm using an adaptive 
Gaussian kernel were compared in this experiment, as shown in Figure 4. In addition, one of the results of registering noisy CT scans and 3D laser scans is shown in Figure 5.

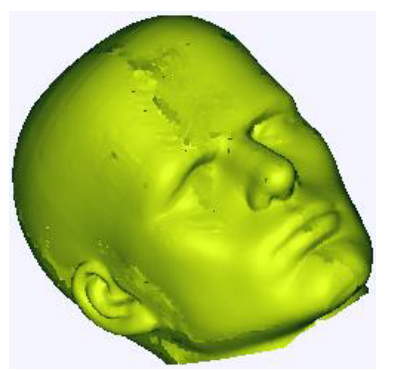

(a)

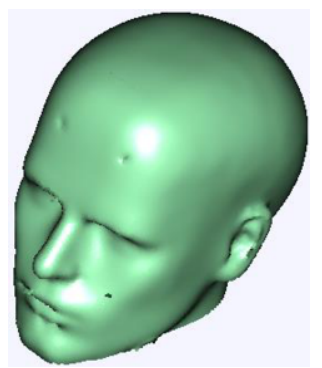

(b)

Fig. 3. Scanning data used in experiments. (a) CT scan with 2,537,960 scanning points. (b) 3D laser scan with 178,268 scanning points.

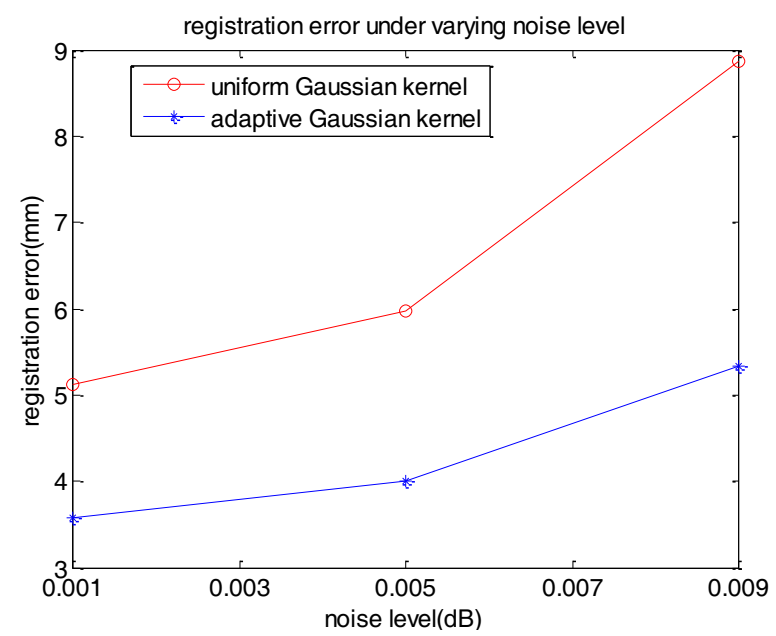

Fig. 4. Comparison of the algorithms using uniform Gaussian kernel and adaptive Gaussian kernel.

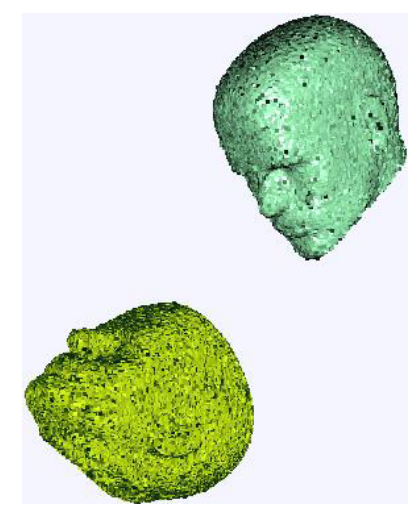

(a)

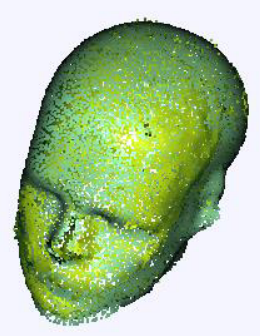

(b)

Fig. 5. Registration result of noisy scans. (a) Two data with $0.005 \mathrm{~dB}$ noise level before registration. (b) The result after automatic registration. 
As seen in the results shown in Fig. 4, for two point sets from different scanning modes, using an adaptive Gaussian kernel can achieve lower registration errors than using a uniform Gaussian kernel. It indicated that an adaptive Gaussian kernel helped generate better approximated surfaces and thus extracted precise principal axes. Meanwhile, when the noise level rose from $0.001 \mathrm{~dB}$ to $0.009 \mathrm{~dB}$, the registration errors increased by approximately 50\% using an adaptive Gaussian kernel, which was less than the increase of $70 \%$ using a uniform Gaussian kernel. Therefore, these results confirmed the robustness of the algorithm proposed in this paper.

It was found that because anesthesia and ventilator tubes are oftentimes in a patient's mouth or nose, geometrical distortions appear in 3D laser scans when comparing them to the preoperative images. Therefore, 3D laser scans with geometrical distortions were simulated and then registered with the preoperative CT scans in six experiments, in which the distortion amplitudes were random for the 3D laser scans. The average error of surface registration for these experiments was $4.22 \mathrm{~mm}$. The registration result of one of the experiments is shown in Figure 6.

As seen in the registration result shown in Figure 6, as well as the average error of these experiments, the registration accuracy of the proposed algorithm was acceptable in the presence of the geometrical distortions. It indicated that the proposed algorithm was robust to the distortions that existed in the pre- or intra-operative scans.

\subsection{Accuracy and runtime of the algorithm}

The proposed algorithm was also compared with the spin image algorithm based on dimension reduction [10], and the comparison results are listed in Table 1. The experiments were conducted under two conditions: in the first, two point sets were scanned with the same device, and in the second condition, they were scanned with different devices. Accuracy and runtime of registration were compared for the two algorithms.

To illustrate the algorithm's feasibility in practical application, partial laser scans and CT scans, as shown in Figure 2, were aligned. It should be noted that the CT scans were manually segmented before extraction of the principal axes to help them correspond with the laser scans. The registration result is shown in Figure 7.

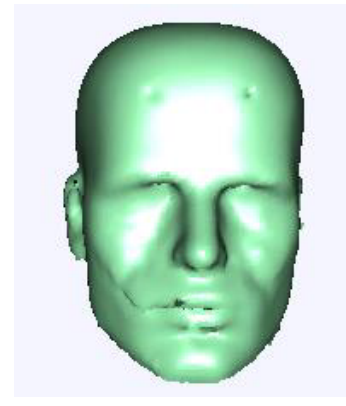

(a)

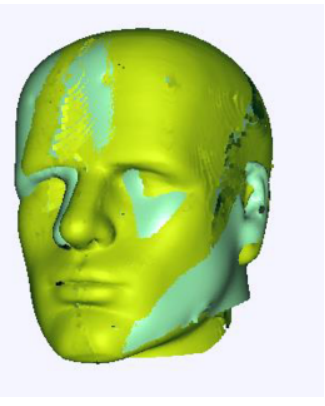

(b)

Fig. 6. Registration result in the presence of geometrical distortions. (a) 3D laser scan with distortions. (b) Result of registering the 3D laser scan in (a) with the CT scan in Figure 3(a). 
Table 1

Comparison of the proposed algorithm to the spin image algorithm

\begin{tabular}{lllll}
\hline \multirow{2}{*}{ Scanning modes } & Proposed algorithm & \multicolumn{3}{l}{ Spin image algorithm } \\
\cline { 2 - 5 } & Accuracy $(\mathrm{mm})$ & Runtime $(\mathrm{s})$ & Accuracy $(\mathrm{mm})$ & Runtime $(\mathrm{s})$ \\
\hline Same & 1.32 & 147 & 1.73 & 270 \\
\hline Different & 4.00 & 168 & 6.42 & 300 \\
\hline
\end{tabular}

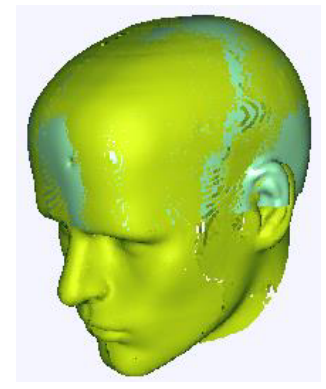

Fig. 7. Registration result of partial laser scan and CT scan from Figure 2.

As seen in the results listed in Table 1, compared to the spin image algorithm based on dimension reductions, the proposed registration algorithm provided higher accuracy and less runtime, especially for two point sets of different scanning modes, which indicated the value of the algorithm in clinical applications.

From the registration result in Figure 7, it was obvious that heuristics was very useful in finding the optimal corresponding centers of translation, especially for incomplete scans.

\section{Conclusion}

Surface registration in clinical applications requires working with scanning data acquired with different devices. This paper presented an automatic coarse registration algorithm based on the principal axes for aligning CT scans with 3D laser scans. The proposed adaptive Gaussian kernel is insensitive to various scanning data and thus improves the principal axes extracted from the approximated surfaces. Additionally, the adopted heuristics is able to determine the corresponding centers of translation accurately. The experimental results confirmed the robustness and efficiency of the proposed algorithm.

\section{Acknowledgement}

This study is partly supported by Project 81101128 and 81271670 sponsored by the National Natural Science Foundation of China, National High Technology Research and Development Program (No. 2012AA02A606), Key Technologies R \& D program of China (No. 2012BAI14B05), Project 12441901600 supported by the Science and Technology Committee of Shanghai Municipality. 


\section{References}

[1] W.K. Pfisterer, S. Papadopoulos, D.A. Drumm, K. Smith and M.C. Preul, Fiducial versus non-fiducial neuronavigation registration assessment and considerations of accuracy, Neurosurgery 63 (2008), 201-207.

[2] C.R. Maurer Jr., J.M. Fitzpatrick, M.Y. Wang, R.L. Galloway Jr., R.J. Maciunas and G.S. Allen, Registration of head volume images using implantable fiducial markers, IEEE Trans. Med. Imaging 16 (1997), 447-462.

[3] M.N. Wang and Z.J. Song, Classification and analysis of the errors in neuronavigation, Neurosurgery 68 (2011), 11311143.

[4] G.C. Sharp, S.W. Lee and D.K. Wehe, ICP registration using invariant features, IEEE Trans. Pattern Anal. Mach. Intell. 24 (2002), 90-102.

[5] J. Salvi, C. Matabosch, D. Fofi and J. Forest, A review of recent range image registration methods with accuracy evaluation, Image and Vision Computing 25 (2007), 578-596.

[6] L. Joskowicz, R. Shamir, M. Freiman, M. Shoham, E. Zehavi, F. Umansky and Y. Shoshan, Image-guided system with miniature robot for precise positioning and targeting in keyhole neurosurgery, Computer Aided Surgery 11 (2006), 181193.

[7] R.R. Shamir, M. Freiman, L. Joskowicz, S. Spektor and Y. Shoshan, Surface-based facial scan registration in neuronavigation procedures: A clinical study, J. Neurosurg. 111 (2009), 1201-1206.

[8] A. Myronenko and X. Song, Point set registration: Coherent point drift, IEEE Trans. Pattern Anal. Mach. Intell. 32 (2010), 2262-2275.

[9] W. Wein, J.Z. Cheng and A. Khamene, Ultrasound based respiratory motion compensation in the abdomen, MICCAI 2008 Workshop on Image Guidance and Computer Assistance for Soft Tissue Interventions 32 (2008), 294.

[10] F.A. Reda, J.H. Noble, R.F. Labadie and B.M. Dawant, Automatic pre-to intra-operative CT registration for imageguided cochlear implant surgery, IEEE Trans. Biomed. Eng. 59 (2012), 3070-3077.

[11] S. Theodoridis and K. Koutroumbas, Feature generation I: Data transformation and dimensionality reduction, in: Pattern Recognition (4th ed.), Academic Press, San Diego, USA, 2008, pp. 326-334.

[12] P. Yang and X. Qian, Direct computing of surface curvatures for point-set surfaces, Proc. Eurographics Symposium on Point-based Graphics, 2007, 29-36. 